\title{
Cytogenetic characterization of the Arabidopsis thaliana natural tetraploid ecotype Warschau stability during in vitro regeneration
}

\author{
Maja Orzechowska $^{1} \cdot$ Sylwia Gurdek $^{1} \cdot$ Dorota Siwinska $^{1} \cdot$ Anna Piekarska-Stachowiak $^{2}$
}

Received: 23 August 2015/Accepted: 6 May 2016/Published online: 17 May 2016

(c) The Author(s) 2016. This article is published with open access at Springerlink.com

\begin{abstract}
The morphological and cytogenetic features of the natural autotetraploid Arabidopsis thaliana ecotype Warschau (Wa-1) were investigated. Most of the Warschau plant organs that were analyzed showed higher size values in comparison with diploid Columbia plants. The tetraploid chromosome number was confirmed by analysis of mitotic metaphase cells and rDNA loci were localized. 35S rDNA loci were present on chromosomes 2 and 4 , while $5 \mathrm{~S}$ rDNA, which is polymorphic among A. thaliana ecotypes, were present on chromosomes 4 and 5. Well-characterized autotetraploid plant material was used for in vitro culture to investigate somaclonal variation. Efficient regeneration through organogenesis was achieved. Most of the plants obtained in vitro exhibited an unchanged ploidy level. Detailed cytogenetic analysis that included chromosome, chromocenters and rDNA signals numbers, revealed the stability of regenerants. Based on these data we recommend the ecotype Warschau as a well-characterized plant material for future investigations on the consequences of polyploidy for the genome.
\end{abstract}

Keywords Arabidopsis · Polyploidy · Somaclonal variation · Fluorescence in situ hybridization

Maja Orzechowska

maja.orzechowska@us.edu.pl

1 Department of Plant Anatomy and Cytology, University of Silesia in Katowice, Jagiellonska 28, 40-032 Katowice, Poland

2 Department of Biophysics and Morphogenesis of Plants, University of Silesia in Katowice, Jagiellonska 28, 40-032 Katowice, Poland

\author{
Abbreviations \\ 2,4-D 2,4-Dichlorophenoxyacetic acid \\ 2iP 6- $(\gamma, \gamma$-Dimethylallylamino) purine \\ B5 Gamborg et al. (1968) medium \\ DAPI 4'-6-Diamino-2-phenyloindole \\ FISH Fluorescence in situ hybridization \\ IAA Indole-3-acetic acid \\ MS Murashige and Skoog (1962) medium \\ NAA $\alpha$-Naphtalene acetic acid \\ rDNA Ribosomal DNA \\ rRNA Ribosomal RNA \\ RT Room temperature
}

\section{Introduction}

Plant in vitro culture is extensively employed in fundamental research and in plant production, conservation and improvement. Although the original idea of in vitro plant propagation was to clone particular genomes, it proven to be a novel source of genetic variability (Larkin and Scowcroft 1981). The nature and origin of somaclonal variation still remains unclear, and therefore it is difficult to regulate the degree of variation with a view to exploring the possibility of regenerating plants with commercially desirable features. A number of factors that influence the level of somaclonal variation have been recognized. In addition to culture conditions, such as type and concentration of growth regulators or the regeneration system, the type of explant has been proven to play an important role (Hao and Deng 2002; Plader et al. 1998). In polysomatic plants, such as Arabidopsis thaliana, an explant can be the source of a preexisting variation due to the presence of endopolyploid cells in the vegetative tissues (Galbraith et al. 1991). A. thaliana, as a model dicot plant, has been widely investigated in 
almost all disciplines of plant biology, including in vitro cultures. Efficient protocols for somatic embryogenesis, organogenesis and transformation for the different ecotypes of A. thaliana have been developed (Akama et al. 1992; Gaj 2001; Valvekens et al. 1988). The ecotype and ploidy level were also taken into account as influential factors in regeneration efficiency and somaclonal variation (Fras and Maluszynska 2003, 2004; Gaj et al. 1997; Zhao et al. 2013). Previously, mostly diploid ecotypes and induced polyploids of $A$. thaliana have been utilized in research. Tetraploid $A$. thaliana plants were usually obtained either via colchicine treatment (Redei 1964; Yu et al. 2009) or after regeneration from in vitro culture (Altmann et al. 1994). The use of induced polyploids is convenient because they can be compared to diploids that have the same genome; however, natural tetraploid accessions can display unique genome composition, as they have existed in natural environment for hundreds of generations. There are only two natural tetraploid accessions of $A$. thaliana that are available in stock centres, both of which have been identified by flow cytometry measurement (Henry et al. 2005; Schmuts et al. 2004). One of these is the Warschau (Wa-1) ecotype, which has shown a high resistance to powdery mildew disease (Adam and Somerville 1996; Schiff et al. 2001). It has been used as a parental accession of triploids and aneuploids, which were widely analyzed for genetic and phenotype variability (Henry et al. 2005, 2007, 2009). Recently, Wa-1 was included in genome-wide association studies of over a hundred A. thaliana lines (Atwell et al. 2010). Nevertheless, there is little information related to the morphology and development of the Warschau ecotype and its karyotype has not been studied in detail to date. There has also been no in vitro culture research involving Wa-1. We consider this natural tetraploid ecotype to be suitable plant material for analyzing the influence of in vitro culture on genome stability. Our previous research showed extensive numerical and structural chromosome rearrangements in the diploid ecotype Columbia (Orzechowska et al. 2013). Artificial autopolyploids of $A$. thaliana have also exhibited variation in chromosome number in callus and in regenerated plants (Fras and Maluszynska 2003). In this paper we examine the effect of genotype on callus induction, shoot regeneration and variation in chromosome number in Wa-1, a natural tetraploid ecotype of A. thaliana.

\section{Materials and methods}

\section{Plant material and morphology observations}

Plants of the Columbia (Col-0) and Warschau (Wa-1) ecotypes were grown in soil in greenhouse with a 16-h day length at a temperature of $21^{\circ} \mathrm{C}$. The plant height, number of siliques per plant and size of leaves were analyzed in ten plants from each ecotype and four rosette leaves from each plant were measured. Silique size, number of seeds per silique, sepals, petals and seed size were analyzed in ten organs of each ecotype. Daily observations were performed in order to assess any differences between the ecotypes in the growth stages lifetime.

The data obtained from the observations of morphology were statistically tested to verify whether there were any significant differences between the plants of Columbia and Warschau ecotypes. The parametric $t$ test for independent samples (for length and width) and the non-parametric U Mann-Whitney test (for other data) were applied. All statistical analyses were conducted using STATISTICA version 10.0 (StatSoft. Inc. 2011).

\section{In vitro culture}

Seeds of the $A$. thaliana plant Warschau ecotype ( $2 n=4 x=20)$ were surface sterilized and placed on a MS medium without growth regulators. Rosette leaves of 3-week-old sterile plants were collected in sterile conditions. Explants, $1 \mathrm{~cm}^{2}$ squares, were cultured on a B5 callus-inducing medium (CIM) (Gamborg et al. 1968) containing $0.5 \mathrm{mg} / \mathrm{L}$ of 2.4-D and $0.05 \mathrm{mg} / \mathrm{L}$ of kinetin. After 6 weeks the calli were transferred onto a shoot-inducing B5 medium (SIM) with $0.15 \mathrm{mg} / \mathrm{L}$ of IAA and $5 \mathrm{mg} / \mathrm{L}$ of $2 \mathrm{iP}$ (Valvekens et al. 1988). The regenerated rosettes and shoots were transferred to a root-inducing B5 medium (RIM) supplemented with $0.125 \mathrm{mg} / \mathrm{L}$ of NAA. The plants and tissue cultures were grown at $21{ }^{\circ} \mathrm{C}$ under a 16-h photoperiod with a light intensity of $70 \mu \mathrm{mol}$ photons $\mathrm{m}^{-2} \mathrm{~s}^{-1}$.

\section{Histology}

To analyze the type of regeneration the callus lumps were fixed after 2, 4, 6 and 8 weeks of culture on SIM in a $37 \%$ paraformaldehyde : acetic acid : $70 \%$ ethanol (0.5:0.5:9) solution. The callus tissue was dehydrated in a series of alcohol and xylene and embedded in paraffin. Semithin sections $(\sim 8 \mu \mathrm{m})$ were cut using a Leica RM2145 microtome and stained with safranin and fast green. The sections were mounted in DPX and analyzed with a Zeiss Axiostar Plus light microscope.

\section{Flow cytometry}

Flow cytometry has been used to analyze endoreduplication pattern and the ploidy levels of the control and regenerated plants. Details of the flow cytometry technique have been described before by Orzechowska et al. (2013). 


\section{Cytogenetic analysis}

The number of chromosomes and chromocenters was analyzed in the flower buds of the control plants and regenerants. The flower buds were pre-treated with $2 \mathrm{mM}$ 8-hydroxyquinoline for $2 \mathrm{~h}$ at RT and $2 \mathrm{~h}$ at $4{ }^{\circ} \mathrm{C}$ and then fixed overnight in a fresh Carnoy's solution (methanol + glacial acetic acid, $3: 1, \mathrm{v} / \mathrm{v})$. The fixed material was stored at $-20{ }^{\circ} \mathrm{C}$ until used. The chromosome preparation procedure was performed as described in Orzechowska et al. (2013). The chromosome preparations were stained with DAPI $(0.5 \mu \mathrm{g} / \mu \mathrm{L})$ and analyzed using an epifluorescence microscope (Olympus). The probes that were used for fluorescence in situ hybridization (FISH) were (1) a 2.3$\mathrm{kb}$ ClaI fragment of the 25S rRNA gene isolated from $A$. thaliana (Unfried and Gruendler 1990), labelled with digoxigenin-11-dUTP (Roche) using a nick translation kit (Roche) according to the manufacturer's protocol and immunodetected with fluorescein conjugated antibodies and (2) a 410-bp a pTa794 fragment of the 5S rDNA isolated from Triticum aestivum (Gerlach and Dyer 1980) and labelled with rhodamine-5-dUTP (Roche) by polymerase chain reaction. The slides that were used for the analysis of chromosome number were re-fixed in a mixture of $99.9 \%$ Et-OH:glacial acetic acid (3:1) for 2-3 min to remove DAPI, rinsed with $99.9 \%$ ethanol, air dried and used for FISH. The FISH experiments were performed following the protocol described in Orzechowska et al. (2013).

\section{Results and discussion}

\section{Characterization of $A$. thaliana Warschau ecotype}

The morphology and growth stages of the tetraploid ecotype Warschau were compared to the diploid Columbia (Col-0) ecotype, which is widely used and well characterized as a model ecotype of A. thaliana (Boyes et al. 2001). The analyses revealed that ten morphological features had higher average values in the Wa-1 plants (Table 1). The most striking differences were related to plant height, the number of side bolts, the size of the flowers and the number of siliques (Fig. 1a-c). The only case in which the diploid Columbia plants showed a statistically higher value than Warschau was for the number of seeds per silique (Table 1), which can suggest a lower fertility of tetraploids (Comai 2005). The pollen of Wa-1 has four colpi, which is typical for A. thaliana tetraploids (Fig. 1d; Altmann et al. 1994). Some differences were also noticed in the plant development stages. Hypocotyls and cotyledons of Col-0 emerged on the fourth day, while in Wa-1 they emerged on the sixth day, and the first rosette leaves emerged 3-4 days later in Wa-1 than in Col-0.
However, the first flower buds were visible in Wa-1 8-12 days earlier than in Col-0. Early flower induction seems to be typical for $A$. thaliana polyploids as it was observed in other tetraploid ecotypes (Karcz et al. 2000).

Analyses of the morphology and development of the Warschau ecotype revealed the typical features that are observed in polyploids. An effect of polyploidy on the developmental rates as well as the overall size of cells, organs and the entire organism is frequently observed in plants (Otto and Whitton 2000). Similar differences were found within diploid plants of the other A. thaliana ecotype Wilna and its induced tetraploids (Karcz et al. 2000).

To date, the tetraploidy of Wa-1 has only been documented by flow cytometry measurements (Henry et al. 2005; Schmuts et al. 2004) and even basic mitotic chromosome observations have not been published. Therefore, cytogenetic analyses of the Warschau ecotype comprised chromosome counts and localization of the rRNA genes. After DAPI staining, 20 chromosomes were observed in the somatic cells of Wa-1 flower buds; however, aneusomaty that has previously been described in the flower buds of Arabidopsis (Orzechowska et al. 2013) was noticed in this case as well. Aneuploid chromosome numbers ranging from 16 to 19 chromosomes were observed in $47 \%$ of the cells analyzed in Wa-1 (Fig. 2a). Localization of the rRNA genes in Wa-1 chromosomes showed eight FISH signals for $35 \mathrm{~S}$ rDNA and eight for $5 \mathrm{~S}$ rDNA (Fig. 3a). The loci of 35S rDNA were localized on chromosomes 2 and 4 . One 5S rDNA locus was present on chromosome 4 and the second on chromosome 5 .

Localization of 35S rRNA genes for Wa-1 chromosomes revealed it is in accordance with 35S rDNA distribution observed for all of the A. thaliana ecotypes analyzed to date (Fransz et al. 1998; Maluszynska and Heslop-Harrison 1991; Murata et al. 1997). However, in the artificial autopolyploid of $A$. thaliana $35 \mathrm{~S}$ rDNA translocation has been described as a part of the mechanism of polyploid genome evolution (Weiss and Maluszynska 2000). 5S rDNA distribution in Wa-1 is similar to observed in the ecotypes Wassilewskija (Fransz et al. 1998) and Wilna (Weiss and Maluszynska 2000). Although, polymorphism of 5S rDNA loci number and localization occurs in A. thaliana and an additional locus is present on chromosome 3 in some ecotypes or even laboratory strains (Fransz et al. 1998). The number of 35S and $5 \mathrm{~S}$ rDNA loci that were observed in Wa-1 corresponded with the doubled number of rDNA loci found in diploid ecotypes. Such a situation occurs frequently in allo- and autopolyploid species, although the elimination of $35 \mathrm{~S}$ and/ or 5S rDNA loci during diploidization is well known (Hasterok et al. 2006; Kolano et al. 2012).

Arabidopsis thaliana is a polysomatic plant so the flow cytometry analysis of the nuclei isolated from Wa-1 young rosette leaves were performed. It showed that the level of 
Table 1 Comparison of the morphological features of the diploid Columbia (Col-0) and the tetraploid Warschau (Wa-1) ecotypes

\begin{tabular}{lrrrl}
\hline Morphological feature & Col-0 & Wa-1 & test & $p$ \\
\hline Plant height (cm) & 25.74 & 30.90 & $\mathrm{t}=-9.83$ & $0.00^{*}$ \\
Average number of side bolts (count) & 0.90 & 2.50 & $\mathrm{Z}=-3.74$ & $0.00^{*}$ \\
Size of rosette leaves (cm) & & & & \\
$\quad$ Length & 2.51 & 2.43 & $\mathrm{t}=0.97$ & 0.33 \\
$\quad$ Width & 1.02 & 1.45 & $\mathrm{t}=-7.44$ & $0.00^{*}$ \\
Size of shoot leaves (cm) & & & \\
Length & 0.95 & 1.34 & $\mathrm{t}=-5.79$ & $0.00^{*}$ \\
$\quad$ Width & 0.38 & 0.53 & $\mathrm{t}=-4.93$ & $0.00^{*}$ \\
Size of sepals (mm) & & & & \\
Length & 1.56 & 2.17 & $\mathrm{t}=-18.99$ & $0.00^{*}$ \\
$\quad$ Width & 0.53 & 0.71 & $\mathrm{t}=-9.67$ & $0.00^{*}$ \\
Size of petals (mm) & & & \\
Length & 1.84 & 2.44 & $\mathrm{t}=11.59$ & $0.00^{*}$ \\
$\quad$ Width & 0.87 & 1.38 & $\mathrm{t}=-26.45$ & $0.00^{*}$ \\
Average size of seeds (mm) & & & & \\
$\quad$ Length & 0.52 & 0.52 & $\mathrm{t}=-0.27$ & 0.79 \\
Width & 0.33 & 0.32 & $\mathrm{t}=0.88$ & 0.39 \\
Average silique length (cm) & 1.02 & 1.07 & $\mathrm{t}=-0.97$ & 0.34 \\
Average number of siliques per plant (count) & 58.60 & 80.40 & $\mathrm{Z}=-3.74$ & $0.00^{*}$ \\
Average number of seeds per silique (count) & 33.90 & 21.40 & $\mathrm{Z}=3.74$ & $0.00^{*}$ \\
\hline
\end{tabular}

$t$ test for independent samples; $Z \mathrm{U}$ Mann-Whitney test

* Statistically significant differences $(p \leq 0.05)$

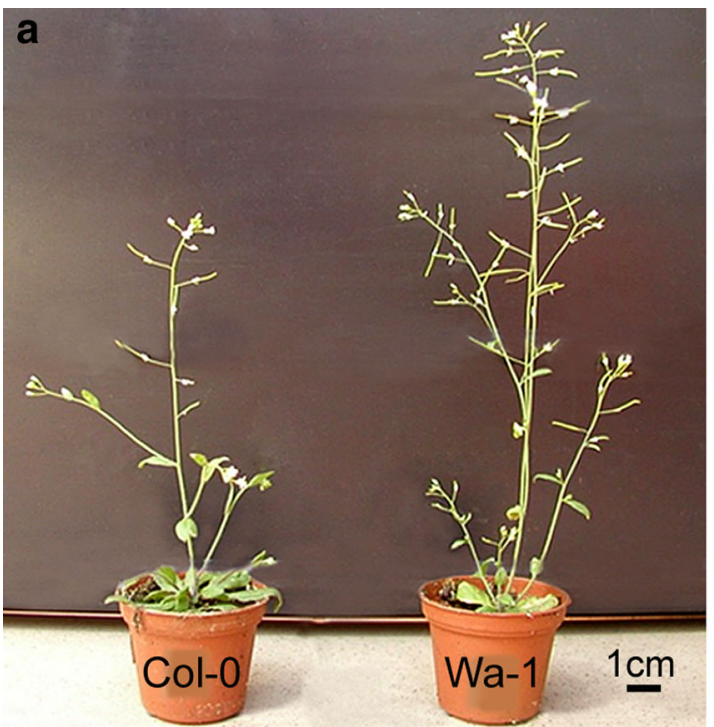

Fig. 1 Comparison of the morphological features of the diploid Col0 and the tetraploid Wa- 1 ecotypes. a Plants of Wa- 1 are higher and have over twice more side bolts than Col-0; b Leaves of Wa-1 have more round shape than Col-0; $\mathbf{c}$ Comparison of flowers size between

endopolyploidy ranged from 4C to $16 \mathrm{C}$. The same range of nuclear DNA content was observed by Fras et al. (2007) in the diploid ecotypes Col and Wilna and in the induced tetraploids of $A$. thaliana ecotype Wilna. It suggest that the
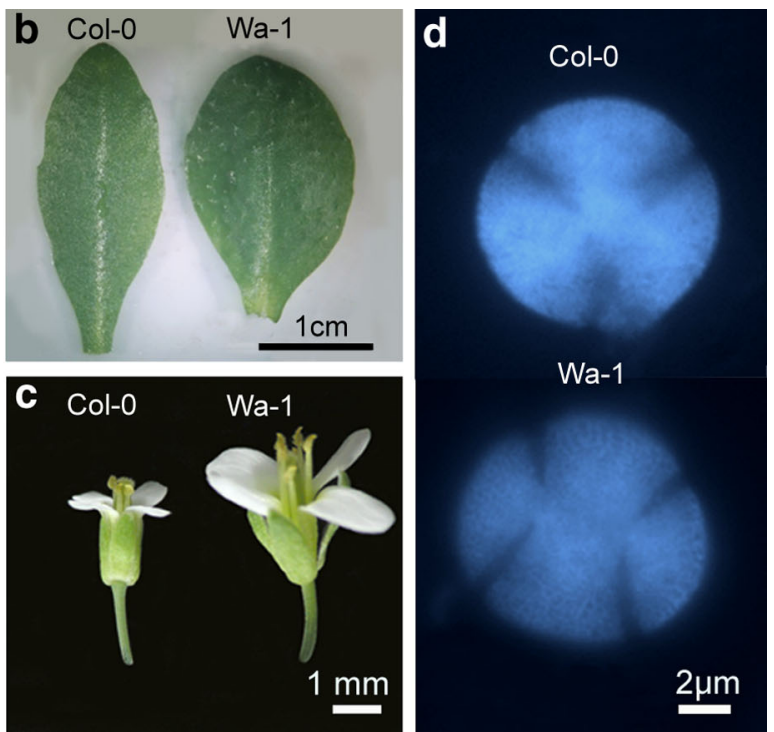

Col-0 and Wa-1; d Autofluorescence of pollen sporodermis in UV light; pollen of Col-0 plant has three colpi, pollen of Wa-1 plant has four colpi, what is typical for tetraploid ecotypes

number of endoreduplication rounds in A. thaliana is not correlated with the plant ploidy level as has been observed, for example, in Lycopersicon esculentum (Smulders et al. 1994). 


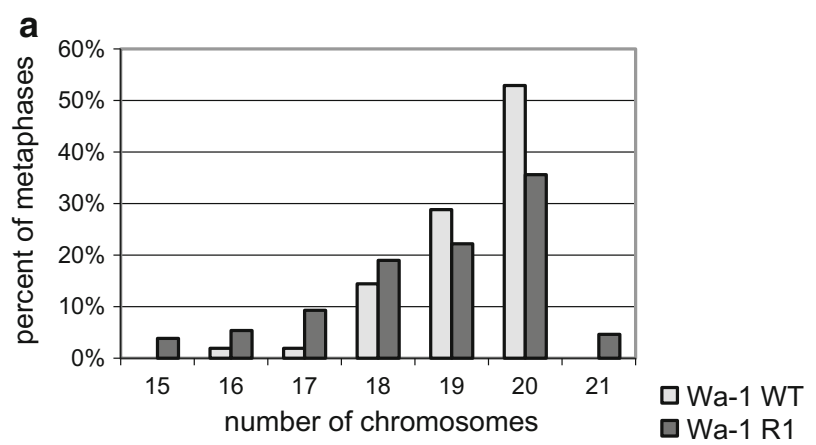

b

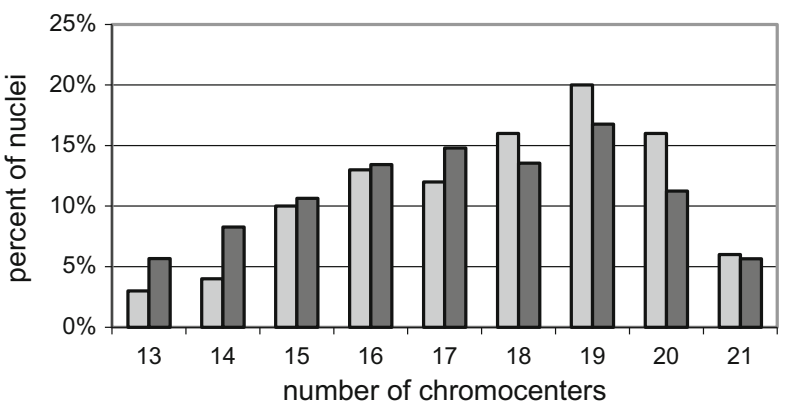

Fig. 2 The percentage of cells displaying varying number of chromosomes (a) and chromocenters (b) in Warschau control plants (Wa-1 WT) and regenerants (Wa-1 R1)
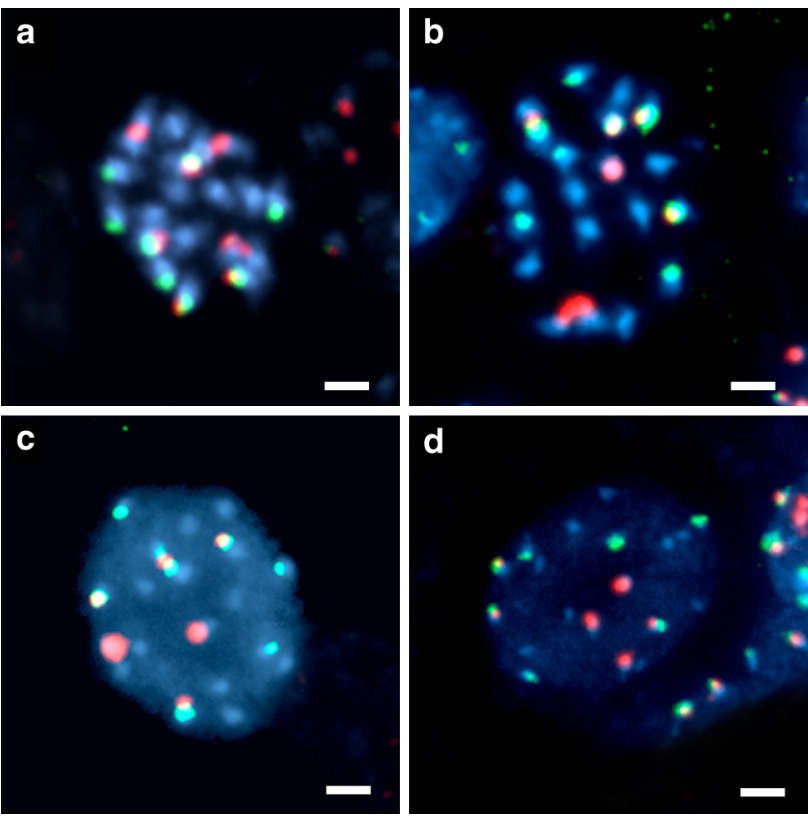

Fig. 3 The number and localization of 35S rDNA and 5S rDNA sites in the chromosomes $(\mathbf{a}, \mathbf{b})$ and interphase nuclei $(\mathbf{c}, \mathbf{d})$ of the $A$. thaliana Wa-1 ecotype. a-c Control plants; b-d Regenerants. Nuclei and chromosomes stained with DAPI (blue fluorescence); $35 \mathrm{~S}$ rDNA labeled with fluorescein (green fluorescence); 5S rDNA labeled with rhodamine (red fluorescence). Bar $1 \mu \mathrm{m}$
The presented data provide good characterization of the natural tetraploid Arabidopsis ecotype Warschau. No abnormalities in morphology or development were observed that could affect the use of the ecotype Wa-1 in in vitro culture and chromosomes has been well characterized which will allow to use it in many research areas, including genome response during in vitro culture.

\section{In vitro regeneration}

The regenerated plants of $A$. thaliana Wa- 1 were obtained after a three-step culture using callus (CIM), shoot (SIM) and root (RIM) inducing media. In Wa-1, $98 \%$ of the leaf explants responded to growth regulators in CIM medium after 6 weeks. Dark green areas appeared on the calluses after their transfer onto SIM and the first leaves were observed in the sixth week. The number of shoots per callus was on average 9.42. The transfer of rosettes onto RIM medium was laborious because of the numerous rosette leaves on the callus tissue. Hence, only $72 \%$ of transferred rosettes developed roots and allowed to obtain mature plants (Fig. 4a). According to this the separation of the callus tissue into pieces of size about $1 \mathrm{~cm}^{3}$ before transfer to the SIM medium is recommended.

The system of a three-step culture has been proven to allow a high degree of regeneration efficiency (Feldmann and Marks 1986; Orzechowska et al. 2013). Comparing regeneration efficiency obtained for Wa- 1 with the A. thaliana diploid ecotypes regenerated via similar systems of in vitro culture the percentage of explants that were capable of organ regeneration is similar, but the number of shoots per callus is much higher in Wa-1. Candela et al. (2001) obtained for leaf explants from 0.07 to 2.01 shoots per explant depending on ecotype and medium composition. The highest frequency of shoots obtained by Zhao et al. (2013) was 5.00 for Wassilewskija ecotype. This result confirms that the ecotype of the explant is an essential factor that influences the regeneration response. The regeneration ability of the callus derived from the leaves of induced $A$. thaliana tetraploids has been $20 \%$ higher than the callus from diploid plants, indicating that the ploidy level of the donor plant is another important factor (Fras and Maluszynska 2003).

Histological investigations of the regeneration process in Wa-1 revealed that regeneration occurs through organogenesis (Fig. 4b); however, Fras and Maluszynska (2003) occasionally observed somatic embryos in the culture of diploid and tetraploid A. thaliana leaf explants with the same shoot inducing medium, as in our experiment. Regeneration through organogenesis has a high efficiency although it has been suggested that it increases the occurrence of somaclonal variation (Pontaroli and Camadro 2005). 
Fig. 4 Regeneration of Wa-1 plants. a Morphology of tetraploid regenerants; b Histology of shoot apical meristem observed in Wa-1 callus on a shoot-inducing medium, a thin section stained with safranin and fast green
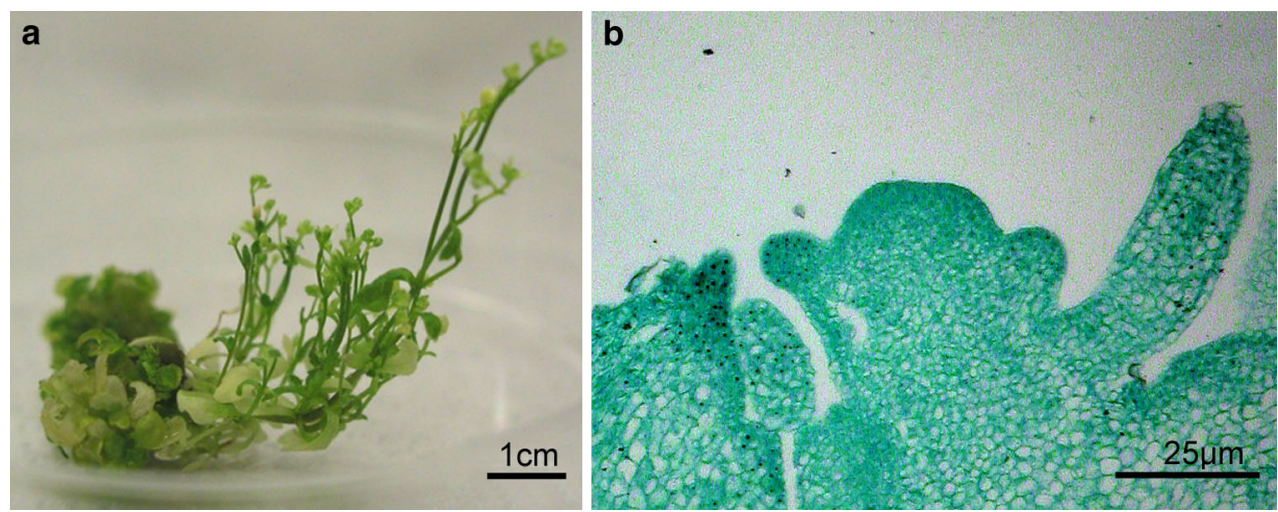

\section{Cytogenetic analysis of regenerants}

Variation in the ploidy level of regenerated plants is the major genetic alteration associated with an in vitro environment and can now be easily detected using flow cytometry (Ochatt et al. 2011). Analysis of $67 \mathrm{Wa}-1$ regenerants revealed that $92.5 \%$ plants were tetraploid, only five $(7.5 \%)$ individuals were octoploid and no diploid or other type of polyploids were observed. Prior studies on A. thaliana diploids indicated the presence of a high proportion of polyploid regenerants (Fras and Maluszynska 2003; Orzechowska et al. 2013), which can be explained by the pre-existing variation in the polysomatic tissues of the explants. On the other hand, more than $50 \%$ of the regenerants that were obtained from the induced tetraploids of A. thaliana, returned to a diploid state (Fras and Maluszynska 2003). These data clearly show that there are significant differences between the ecotypes in their response during in vitro culture, and that the origin of the polyploid donor plant (natural or artificial) can influence the stability of the regenerated specimens.

In addition to variations in the ploidy level, aneuploid chromosome number is another main cause of somaclonal variation. In the Wa-1 regenerants (Wa-1 R1), 312 metaphases from flower buds were analyzed. As aneuploid cells were also observed in Wa-1 plants that had been grown from seeds (Wa-1 WT), the results obtained for the regenerants were compared with data from Wa-1 WT as the control (Fig. 2). The range of chromosome numbers was wider in the plants that had been obtained in vitro. Compared to the control, metaphases with 20 chromosomes had a lower frequency (35\%) and metaphases with 15 and 21 chromosomes were additionally observed (Fig. 2a). Since the number of dividing cells is often limited in callus tissue or in individual regenerants, well-visible chromocenters in the interphase nuclei that correspond to the chromosome number can be used in the case of A. thaliana (Fransz et al. 2002). 1608 interphase nuclei from Wa1-R1 flower buds were analyzed. The differences between the control and regenerated plants were much smaller than in case of metaphase chromosomes, probably due to much higher number of cells analyzed (Fig. 2b). The nuclei that had 19 chromocenters were most frequent in both the control and regenerated plants, and had a lower value $(16.7 \%)$ for the plants that had been regenerated in vitro. For more detailed cytogenetic analysis, fluorescence in situ hybridization with rDNA probes was performed on the chromosome preparations of the best quality. The number of the hybridization signals of $35 \mathrm{~S}$ and $5 \mathrm{~S}$ rDNA was analyzed in 44 metaphases and 100 interphase nuclei from seven regenerated plants and in the control material. The number of both types of rDNA loci in the cells with 20 chromosomes equalled eight in the regenerants as well as in the control plants (Fig. 3b). In the aneuploid metaphases, a random elimination of chromosomes was revealed based on the chromosome identification after FISH, which is congruent with the previous reports (Orzechowska et al. 2013).

The number of 5S rDNA and 35S rDNA hybridization signals per interphase nucleus in control $A$. thaliana plants of Warschau ecotype ranged from three to nine. Number of signals observed in the Warschau regenerants was from three to ten signals per nucleus (Fig. 3c-d). In the control and regenerated plants nuclei with six and seven 35S rDNA signals were most frequent. Analysis of the 5S rDNA signals revealed that the nuclei with eight signals were most frequent in control plant and nuclei with seven signals dominate in regenerants (Fig. 5).

In general, the lower number of rDNA hybridization signals was observed in interphase nuclei than in metaphase chromosomes. It might be explained by the association of chromosomes that have rDNA loci. A high percentage of the homologous associations of chromosome 2 has been described in the diploid nuclei of A. thaliana (Fransz et al. 2002). This type of association has also been detected in artificial A. thaliana autotetraploids (Weiss and Maluszynska 1998). On the other hand the maximum number of $35 \mathrm{~S}$ rDNA and 5S rDNA per nucleus should be eight according to localization of these sequences on mitotic chromosomes; yet, a number of rDNA signals exceeding eight was observed in the control and regenerated plants of Wa-1 ecotype. This 


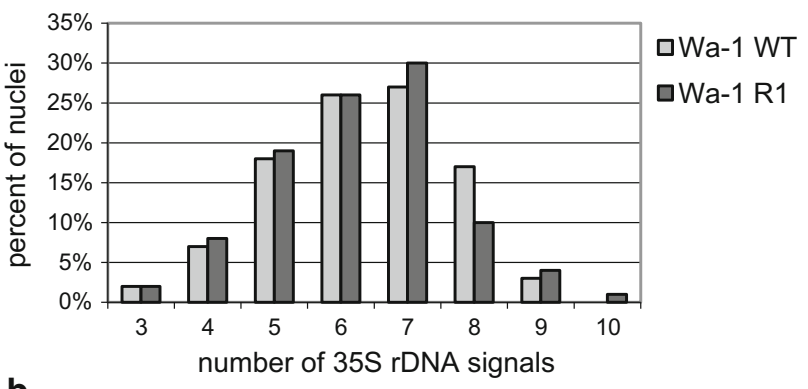

b

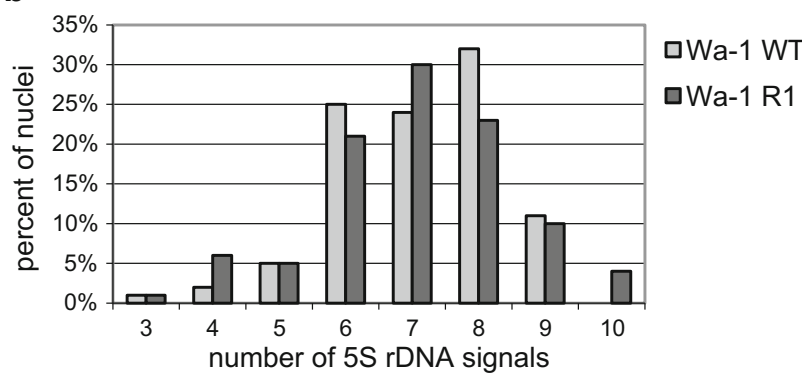

Fig. 5 Distribution of the number of hybridization signals of $35 \mathrm{~S}$ rDNA (a) and 5S rDNA (b) per nucleus in the control (Wa-1 WT) and in vitro regenerated (Wa-1 R1) plants

can be attributed to the fact that some cells of the developing flowers, such as the tapetum, undergo endopolyploidization (Weiss and Maluszynska 2001). The decondensation of rDNA during the interphase can also result in more dispersed hybridization signals.

\section{Conclusions}

Our research revealed the stability of the natural autotetraploid A. thaliana Warschau ecotype during in vitro regeneration. In vitro culture conditions are considered to be a highly mutagenic environment, which can prompt wide genomic changes at different levels of genome organization. Previous in vitro studies of diploid and induced tetraploid ecotypes of $A$. thaliana indicated a considerable rate of somaclonal variation (Fras and Maluszynska 2003, 2004; Orzechowska et al. 2013). As the high regeneration efficiency was achieved and in vitro culture conditions influenced the Wa-1 chromosome number and structure to a marginal degree, we recommend this ecotype as a suitable material for various types of research related to the polyploid genome structure and evolution in vitro as well as in vivo.

Open Access This article is distributed under the terms of the Creative Commons Attribution 4.0 International License (http://crea tivecommons.org/licenses/by/4.0/), which permits unrestricted use, distribution, and reproduction in any medium, provided you give appropriate credit to the original author(s) and the source, provide a link to the Creative Commons license, and indicate if changes were made.

\section{References}

Adam L, Somerville SC (1996) Genetic characterization of five powdery mildew disease resistance loci in Arabidopsis thaliana. Plant J 9:341-356

Akama K, Shiraishi H, Ohta S, Nakamura K, Okada K, Shimura Y (1992) Efficient transformation of Arabidopsis thaliana: comparison of the efficiences with various organs, plant ecotypes and Agrobacterium strains. Plant Cell Rep 12:7-11

Altmann T, Damm B, Frommer WB, Martin T, Morris PC, Schweizer D, Willmitzer L, Schmidt R (1994) Easy determination of ploidy level in Arabidopsis thaliana plants by means of pollen size measurement. Plant Cell Rep 13(11):652-656

Atwell S, Huang YS, Vilhjálmsson BJ et al (2010) Genome-wide association study of 107 phenotypes in Arabidopsis thaliana inbred lines. Nature 465:627-631

Boyes DC, Zayed AM, Ascenzi R, McCaskill AJ, Hoffman NE, Davis KR, Görlach J (2001) Growth stage-based phenotypic analysis of Arabidopsis: a model for throughput functional genomics in plants. Plant Cell 13:1499-1510

Candela M, Velázquez I, De la Cruz B, Sendino AM, De la Peña A (2001) Differences in in vitro plant regeneration ability among four Arabidopsis thaliana ecotypes. In Vitro Cell Dev Biol Plant 37:638-643

Comai L (2005) The advantages and disadvantages of being polyploid. Nat Rev Genet 6:836-846

Feldmann KA, Marks MD (1986) Rapid and efficient regeneration of plants from explants of Arabidopsis thaliana. Plant Sci 47:63-69

Fransz P, Armstrong S, Alonso-Blanco C, Fischer TC, Torres-Ruiz RA, Jones G (1998) Cytogenetics for the model system Arabidopsis thaliana. Plant J 13:867-876

Fransz P, Hand de Jong J, Lysak M, Ruffini Castiglione M, Schubert I (2002) Interphase chromosomes in Arabidopsis are organized as well defined chromocenters from which euchromatin loops emanate. Proc Nat Acad Sci 99:14584-14589

Fras A, Maluszynska J (2003) Regeneration of diploid and tetraploid plants of Arabidopsis thaliana via callus. Acta Biol Crac Ser Bot 45(2): 145-152

Fras A, Maluszynska J (2004) The correlation between the chromosome variation in callus and genotype of explants of Arabidopsis thaliana. Genetica 121:145-154

Fras A, Juchimiuk J, Siwinska D, Małuszyńska J (2007) Cytological events in explants of Arabidopsis thaliana during early callogenesis. Plant Cell Rep 26:1933-1939

Gaj MD (2001) Direct somatic embryogenesis as a rapid efficient system for in vitro regeneration of Arabidopsis thaliana. Plant Cell Tissue Organ Cult 64:39-46

Gaj MD, Ledwon U, Gaj M (1997) Evaluation of plant regeneration ability in culture of immature embryos of different Arabidopsis thaliana (L.) Heynh. Genotypes. Acta Biol Sil 31:66-73

Galbraith DW, Harkins KR, Knapp S (1991) Systemic endopolyploidy in Arabidopsis thaliana. Plant Physiol 96:985-989

Gamborg OL, Miler RA, Ojima K (1968) Nutrient requirement of suspension cultures of soybean root cells. Exp Cell Res 50:151-158

Gerlach WL, Dyer TA (1980) Sequence organization of the repeating units in the nucleus of wheat which contain 5S rRNA genes. Nucleic Acids Res 11:4851-4865

Hao Y-J, Deng X-X (2002) Occurrence of chromosomal variations and plant regeneration from long-term-cultured citrus callus. In Vitro Cell Dev Biol Plant 38:472-476

Hasterok R, Wolny E, Hosiawa M, Kowalczyk M, Kulak-Ksiazczyk S, Ksiazczyk T, Hennen WK, Maluszynska J (2006) Comparative analysis of rDNA distribution in chromosomes of various species of Brassicaceae. Ann Bot 97:205-2016 
Henry IM, Dilkes BP, Young K, Watson B, Wu H, Comai L (2005) Aneuploidy and genetic variation in the Arabidopsis thaliana triploid response. Genetics 170:1979-1988

Henry IM, Dilkes BP, Comai L (2007) Genetic basis for dosage sensitivity in Arabidopsis thaliana. PLoS Genet 3:e70

Henry IM, Dilkes BP, Tyagi AP, Lin HY, Comai L (2009) Dosage and parent-of-origin effects shaping aneuploidy swarms in $A$. thaliana. Heredity 103:458-468

Karcz J, Weiss H, Maluszynska J (2000) Structural and embryological studies of diploid and tetraploid Arabidopsis thaliana (L.) Heynh. Acta Biol Crac 42(2):113-124

Kolano B, Tomczak H, Molewska R, Jellen EN, Maluszynska J (2012) Distribution of $5 \mathrm{~S}$ and $35 \mathrm{~S}$ rRNA gene sites in 34 Chenopodium species (Amaranthaceae). Bot J Linn Soc 170:220-231

Larkin PJ, Scowcroft WR (1981) Somaclonal variation: a novel source of variability from cell cultures for plant improvement. Theor Appl Genet 60:197-214

Maluszynska J, Heslop-Harrison JS (1991) Localization of tandemly repeated DNA sequences in Arabidopsis thaliana. Plant $\mathrm{J}$ $1: 159-166$

Murashige T, Skoog F (1962) A revised medium for rapid growth and bioassays with tobacco tissue cultures. Physiol Plantarum 15:473-497

Murata M, Heslop-Harrison JS, Motoyoshi F (1997) Physical mapping of the 5S ribosomal RNA genes in Arabidopsis thaliana by multi-color fluorescence in situ hybridization with cosmid clones. Plant J 12:31-37

Ochatt SJ, Patat-Ochatt EM, Moessner A (2011) Ploidy level determination within the context of in vitro breeding. Plant Cell Tissue Organ Cult 104:329-341

Orzechowska M, Stępień K, Kamińska T, Siwińska D (2013) Chromosome variations in regenerants of Arabidopsis thaliana derived from 2- and 6-week-old callus detected using flow cytometry and FISH analyses. Plant Cell Tissue Organ Cult 112:263-273

Otto SP, Whitton J (2000) Polyploid incidence and evolution. Annu Rev Genet 34:401-437

Plader W, Malepszy S, Burza W, Rusinowski Z (1998) The relationship between the regeneration system and genetic variability in the cucumber (Cucumis sativus L.). Euphytica 103:9-15
Pontaroli AC, Camadro EL (2005) Somaclonal variation in Asparagus officinalis plants regenerated by organogenesis from long term callus cultures. Gen Mol Biol 28(3):423-430

Redei GP (1964) Crossing experiments with polyploids. Arabidopsis Inf Serv 1:13

Schiff CL, Wilson IW, Somerville SC (2001) Polygenic powdery mildew disease resistance in Arabidopsis thaliana: quantitative trait analysis of the accession Warschau-1. Plant Pathol 50:690-701

Schmuts H, Eister AM, Horres R, Bachmann K (2004) Genome size variation among accessions of Arabidopsis thaliana. Ann Bot 93:317-321

Smulders MJM, Rus-Kortekaas W, Gilissen LJW (1994) Development of polysomaty during differentiation in diploid and tetraploid tomato (Lycopersicon esculentum). Plant Sci 97:53-60

Unfried I, Gruendler P (1990) Nucleotide sequence of the 5.8S and 25S rRNA genes and the internal transcribed spacers from Arabidopsis thaliana. Nucleic Acids Res 18:4011

Valvekens D, Van Montagu M, Van Lijsebettens M (1988) Agrobacterium tumefaciens-mediated transformation of Arabidopsis thaliana root explants by using kanamycin selection. Proc Natl Acad Sci USA 85:5536-5540

Weiss H, Maluszynska J (1998) Cytogenetic investigations of different types of cells from flower budds of Arabidopsis thaliana (L.) Heynh. In: Maluszynska J (ed) Plant cytogenetics, Prace Naukowe Uniwersytetu Śląskiego, pp 195-200

Weiss H, Maluszynska J (2000) Chromosomal rearrangement in autotetraploid plants of Arabidopsis thaliana. Hereditas 133:255-261

Weiss H, Maluszynska J (2001) Molecular cytogenetic analysis of polyploidisation in the anther tapetum of diploid and autotetraploid Arabidopsis thaliana plants. Ann Bot 87:729-735

Yu Z, Haage K, Strei VE, Gierl A, Torres Ruiz RA (2009) A large number of tetraploid Arabidopsis thaliana lines, generated by a rapid strategy, reveal high stability of neo-tetraploids during consecutive generations. Theor Appl Genet 118:1107-1119

Zhao XY, Su YH, Zhang CL, Wang L, Li XG, Zhang XS (2013) Differences in capacities of in vitro organ regeneration between two Arabidopsis ecotypes Wassilewskija and Columbia. Plant Cell Tissue Organ Cult 112:65-74 